\title{
BMJ Open Understanding the importance of therapeutic relationships in the development of self-management behaviours during cancer rehabilitation: a qualitative research protocol
}

\author{
Wendy M Wilkinson, ${ }^{1,2,3}$ Jaynie Rance, ${ }^{4}$ Deborah Fitzsimmons ${ }^{4}$
}

To cite: Wilkinson WM, Rance J, Fitzsimmons D. Understanding the importance of therapeutic relationships in the development of selfmanagement behaviours during cancer rehabilitation: a qualitative research protocol. BMJ Open 2017;7:e012625. doi:10.1136/bmjopen-2016012625

- Prepublication history for this paper is available online. To view these files please visit the journal online (http://dx.doi.org/10.1136/ bmjopen-2016-012625).

Received 12 May 2016 Revised 10 October 2016 Accepted 12 October 2016

\footnotetext{
${ }^{1}$ Abertawe Bro Morgannwg University Health Board, Port Talbot, UK ${ }^{2}$ Wales Cancer Network ${ }^{3}$ Swansea University, Swansea, UK ${ }^{4}$ College of Human and Health Sciences, Swansea University, Swansea, UK
}

Correspondence to Wendy M Wilkinson; 746345@swansea.ac.uk

\begin{abstract}
Introduction: Cancer is a growing health, social and economic problem. 1 in 3 people in the UK will develop cancer in their lifetime. With survival rates rising to over $50 \%$, the long-term needs of cancer survivors are of growing importance. Cancer rehabilitation is tailored to address the physical or psychosocial decline in ability to engage in daily activities. Its use is supported by highquality international, multicentre research. Incorporating strategies for self-management behaviour development into rehabilitation can prepare individuals for cancer survivorship. However, healthcare professionals will need to adjust their therapeutic interactions accordingly. Research is yet to clarify the impact of the therapeutic relationship on rehabilitation outcomes in cancer. This study aims to explore the impact of therapeutic relationships on self-management behaviours after cancer.
\end{abstract}

Methods and analysis: This qualitative study aims to understand cancer rehabilitation participants' beliefs regarding the importance of therapeutic relationships in developing self-management behaviours. A sample representative of a local cancer rehabilitation cohort will be asked to complete a semistructured interview to identify their perspectives on the importance of therapeutic relationships in cancer rehabilitation. Data obtained from the interviews will be analysed, coded and entered into a Delphi questionnaire for circulation to a local cancer rehabilitation population to determine if the views expressed by the interviewees are supported by group consensus.

Ethics and dissemination: This study was approved by Wales Research Ethics Committee 6 (15/WA/0331) in April 2016. Findings will be disseminated through the first author's doctoral thesis; peer-reviewed journals; local, national and international conference presentations; and public events involving research participants and the general public.

\section{INTRODUCTION}

The increasing incidence of cancer is a growing health, social and economic problem. Current estimates expect as many

\section{Strengths and limitations of this study}

- This study starts to explore how therapeutic relationships impact on survivorship, as perceived by cancer rehabilitation participants.

- Cancer rehabilitation participants will inform the design of future cancer services as experts in their experience.

- This foundation study will be unlikely to offer guidance on how to quantify or measure the specific impact of therapeutic relationships.

as one in three people in the UK to develop cancer in their lifetime. ${ }^{1}$ Advancements in screening programmes and treatments have improved 5-year survival rates to over $50 \%$ for some cancers. ${ }^{2}$ A joint Department of Health, Macmillan Cancer Support and NHS Improvement ${ }^{3}$ report forecasts a possible 3\% annual increase in the size of this population with estimation that up to 3 million people in the UK living with a cancer diagnosis by 2030. This makes the long-term needs of cancer survivors a growing importance from a healthcare and social perspective.

\section{Cancer rehabilitation}

Cancer rehabilitation is a complex set of interventions, tailored to meet the needs of individuals who experience a decline in their physical or psychosocial ability to undertake daily activities or life roles at any point following a diagnosis. ${ }^{45}$ Cancer rehabilitation services should be multidisciplinary, focus on meeting the functional impact of cancer and cancer treatments, and aim to optimise individuals' participation in daily activities and life roles. ${ }^{6}$ Allied health professions (AHP), including occupational therapy (OT), physiotherapy, dietetics and speech and language therapy, play key roles in the delivery of cancer rehabilitation programmes. 
The last decade has seen a rapidly expanding programme of high-quality research into cancer rehabilitation. This research portfolio has demonstrated improvements in physical and psychosocial outcomes following cancer rehabilitation. ${ }^{7-11}$

Key opportunities for cancer rehabilitation to impact in improving outcomes for people with cancer include prehabilitation or preparing people for diagnostic investigations or treatment, ${ }^{12-14}$ supporting fluctuations in function throughout and facilitating recovery after treatment ${ }^{14}$ and supporting self-management thereby preparing individuals for cancer survivorship. ${ }^{15}$

\section{Self-management}

There is an increasing body of research guiding selfmanagement interventions for a range of long-term health conditions including cancer. ${ }^{16}$ Much of this research is focused on the impact of patient education, skills development and self-monitoring interventions on enabling people to take control of their health. ${ }^{17}$ Using a self-management approach within a clinical context moves away from traditional rehabilitation service delivery models. Self-management expects a greater level of active participation by healthcare recipients. It also demands an adjustment to the style of therapeutic interactions by healthcare professionals. ${ }^{18}$

\section{Therapeutic relationships}

Using therapeutic relationships to support care delivery aimed at improving treatment outcomes is not unique in OT. There is also evidence supporting therapeutic relationships in other professions, including nursing ${ }^{19}$ and psychotherapy. $^{20}$

In addition to achieving goal-related physical rehabilitation outcomes, ${ }^{21}$ additional key benefits of adopting this approach in practice are the potential to facilitate the development of confidence and self-esteem by reducing anxiety. ${ }^{22}$

In OT, the therapeutic relationship is considered one of the most significant dimensions of treatment interactions $;^{23}$ ideally built through a reflexive process whereby healthcare professionals consciously engage personal attributes and behaviours to develop therapeutic relationships with patients to optimise treatment outcomes.

Although research to date cannot provide clear associations between the impact of the therapeutic relationship on psychosocial outcomes in cancer $^{24}$ due to complexity of these relationships, it does form a solid foundation from which to further look into how this powerful tool might better support self-management for those who are learning to live with or after cancer.

\section{Theoretical framework}

Self-determination theory (SDT) is a person-centred behaviour change model which encourages healthcare professionals to focus on supporting the development of personal autonomy for patient self-managing long-term conditions. Within this framework, the idea of relatedness (identifiable as the therapeutic relationship) can be considered a key mechanism by which change is achieved. $^{25}$ Despite evidence supporting SDT as an effective model in healthcare, $\mathrm{Ng}$ et $a l^{26}$ call for more research into the mechanisms that effect health behaviour change.

Boger $e t a l^{27}$ highlight therapeutic relationships with healthcare professionals, as part of a broader positive network, as a fundamental element in providing effective self-management support. This relationship can promote knowledge, skills, development of identity and independence commonly associated with optimal selfmanagement outcomes, and can be a key feature in people feeling enabled to manage their own care.

\section{METHODS}

Objectives

The objectives of this study are to:

- Describe therapeutic relationships as experienced by people affected by cancer during rehabilitation.

- Identify participants' perspective of potential barriers and facilitators to effective therapeutic relationships during cancer rehabilitation.

- Understand the importance of the therapeutic relationship during cancer rehabilitation in relation to achieving participant-driven outcomes.

\section{Setting}

This qualitative study aims to explore the experiences and perceptions of participants connected with a cancer rehabilitation service located in South West Wales, UK. This service is provided by specialist oncology occupational therapists and physiotherapists based in secondary care, who work closely with specialist oncology medical, nursing, dietetics and speech and language therapy teams. The cancer rehabilitation service links with community, leisure and voluntary sector organisations across South West Wales to ensure participants have access to a holistic programme of physical, emotional and social support. The Health Board, within which this team sits, hosts the South West Wales Cancer Centre, which is responsible for providing specialist cancer services for residents of the local Health Board and one adjacent Health Board. The cancer rehabilitation service provides inpatient rehabilitation to the local oncology/haematology ward, and outpatient rehabilitation for this population.

This study focuses on the outpatient programme, which is free of charge to participants and offers a holistic needs assessment on first contact and at 12 weeks following individual and/or group rehabilitation. This service is available to individuals with all tumour sites, including blood cancers; and can be accessed by self or professional referral before, during and after surgical or oncological treatment. This includes availability for people presenting with long term or late-onset consequences of treatment. See figure 1 for tumour site 
Cancer Rehabilitation Population Composition by Tumour Site in

Relation to Reported Incidence (WCISU 2016).

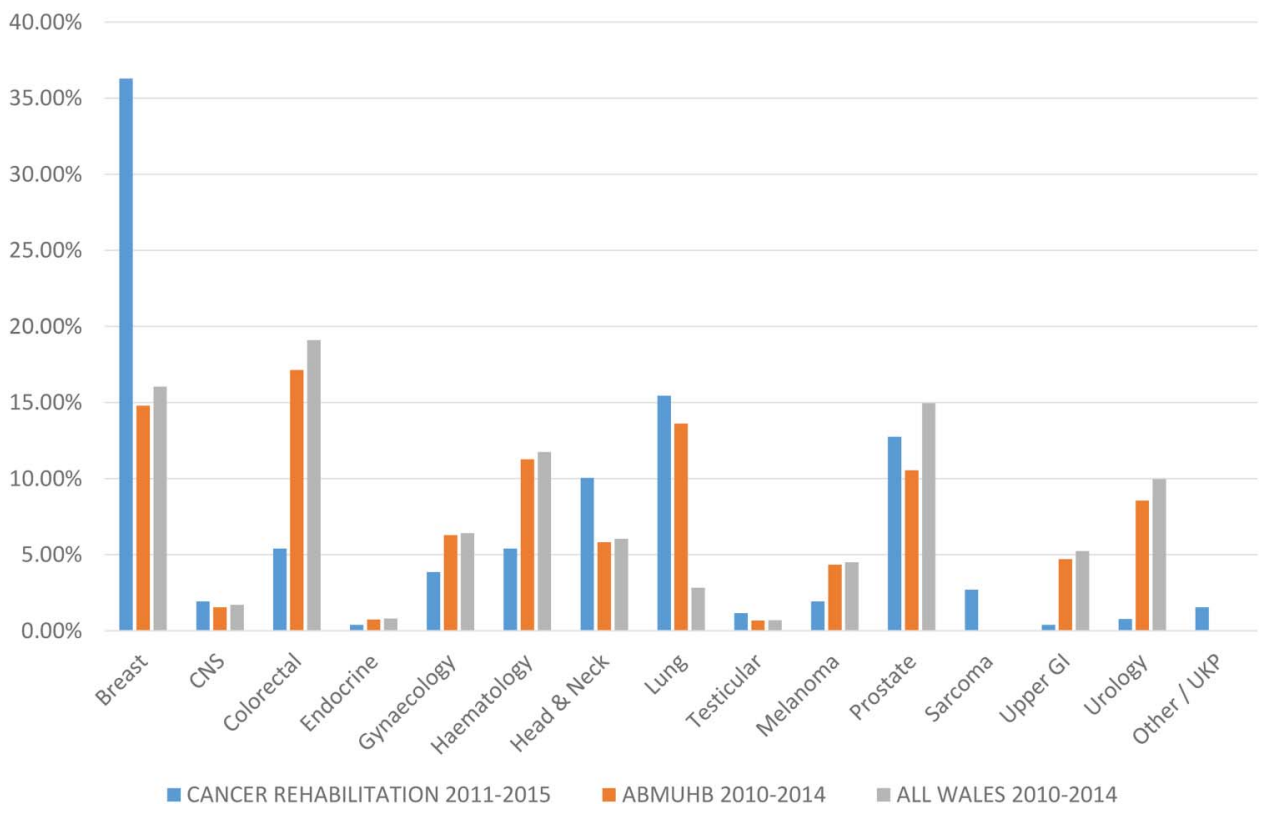

Figure 1 Relative tumour site profile of cancer rehabilitation population (2011-2015).

profile of cancer rehabilitation population from 2011 to 2015.

\section{Participant recruitment}

All people referred to and recorded on the cancer rehabilitation service database from 2011 to 2015 will be eligible to take part in this research. The cancer rehabilitation service does not provide treatment for children. Therefore, eligible research participants would include persons aged 18 years or older, having been affected by cancer of any type, at any disease stage and having completed or be in the process of participating in the local cancer rehabilitation programme (see figure 2 for details).

Recruitment to the semistructured interviews and Delphi questionnaire will follow the procedure outlined in the study overview (see figure 3). An initial list of potential research participants from the service referral database will be cross-checked against a Health Board patient information management system, to ensure that up-to-date contact details are available and ensure that undue distress is not caused by contacting the households of individuals who have not survived their cancer. Eligible participants will be posted a research invitation and preconsent form, allowing time to consider participation in the study. On return of the preconsent form using a reply paid envelope, names and contact details will be held on a password-protected database, accessed by the research team only. This will be required for the practical purposes of providing supplemental information or explanation of the study, to answer any questions, complete demographic screening questions and register participant preference for research activity, arranging of face-to-face interviews and recording the return rate from each round of questionnaires.

A demographic data collection tool, based on the National Cancer Patient Experience Survey, ${ }^{28}$ will be used to identify sample age, sex, ethnicity, highest level of education and main employment status at the time of starting rehabilitation. In addition to this, data will be collected relating to any non-cancer long-term conditions, time since first cancer treatment, whether participants have been treated for one or more cancer diagnoses and type of cancer treatment/disease responses status at the time of rehabilitation.

Following a period of 4 weeks, a second mail out will take place to participants who are yet to have responded. If no further response has been received from potential participants within the following 4 weeks, non-consent will be assumed. These individuals will not be approached again.

\section{Participant choice}

Participant collaboration in service development has been highly valued by the local cancer rehabilitation service from its inception in 2008. In an attempt to maintain this collaborative philosophy throughout the research, the study design will offer participants a choice whether they would like to take part in one semistructured interview and/or up to three Delphi questionnaires.

Ideally, all participants who identify a preference to participate in semistructured interviews will be accommodated. However, in the event that there are more volunteers for the semistructured interviews than are required, participants will be advised during screening 


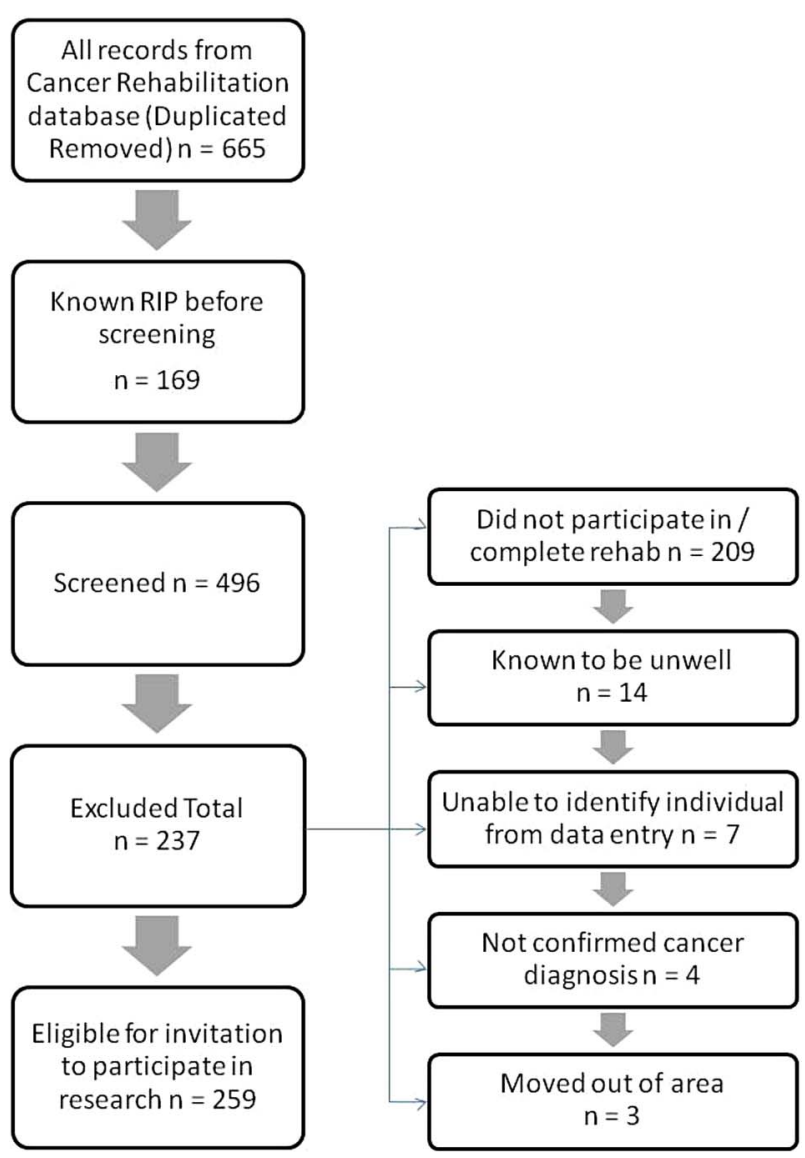

Figure 2 Sampling procedure.

that the interview cohort will be selected from volunteers to provide best fit representation of variables, including tumour site, disease stage, sex and age by the variables' percentage proportion of the total local cancer rehabilitation population. Those participants who indicate a preference for interview, but are not selected, will be asked to consider taking part in the Delphi Technique in order to continue their involvement in the study.

\section{Data collection}

Data collection for this study will take place in two stages. The first stage will include semistructured interviews. The second stage will use a Delphi Technique.

\section{Semistructured interviews}

Semistructured interviews will offer local cancer rehabilitation participants the opportunity to describe their experiences of therapeutic relationships with local practitioners. These data will inform the first round of themes for the Delphi questionnaires. This is aimed to reduce the potential for value judgements to be imposed on the research by the principal investigator. It is estimated that 20 interview participants can be selected to form a representational sample of the local cancer rehabilitation population. The research team reserves the decision to cease interviewing before this quota is reached if data saturation occurs. ${ }^{29}$

A semistructured interview schedule (see table 1) will be piloted and then used to facilitate face-to-face interviews. The interviews will be conducted by the principal investigator who is a member of the specialist oncology therapy team which provides a local cancer rehabilitation programme. A participant briefing will precede each interview, during which informed consent will be confirmed for audio-recording of the interview. It is anticipated that the interviews will last no longer than $60 \mathrm{~min}$, and will take place in an agreed NHS, cancer charity or other location offering a suitably quiet, comfortable and confidential environment. A debriefing opportunity will follow each interview, to ensure that the research participant is aware of the purpose of, and proposed use of the interview content; provide support and signposting to appropriate services if the participant found the interview upsetting.

The interview transcripts will be analysed by the principal investigator using the content analysis framework offered by Graneheim and Lundman. ${ }^{30}$ Each interview transcript will be read, identifying meaning themes including words, phrases and sentences relating to the therapeutic relationships in cancer rehabilitation. These will then be condensed and grouped into themes. It will be these themes which are used to design the first Delphi questionnaire rather than professionals' preconceived codes. $^{31}$

In order to cross-check data analysis to ensure data quality, consistency in approach and transparency of analytical decision-making, ${ }^{29} 20 \%$ of the interview transcripts will be analysed by a second reviewer. A third reviewer will be used where needed to undertake a further analysis and used if thematic discrepancies occur between the first and second reviewer.

\section{Reflexivity}

Stanley and Nayar ${ }^{32}$ advise a reflexive approach to managing the relationship between the researcher and the research participants. Maintaining a research journal, which articulates the experiences of the practitionerresearcher relationship with study participants, is one method which will be used to ensure study rigour. This will explore the advantages and disadvantages of having a prior therapeutic relationship with research participants on eliciting accurate research data which is a true reflection of the participants' beliefs. This journal will also articulate the reasoning behind decision-making during data analysis.

In addition to this, the principal investigator is skilled in recognising if the research relationship runs the risk of transitioning to a therapeutic relationship as a result of rapport building. Redirecting the activity back to the research topic, or sensitively terminating the research activity and offering aftercare will ensure participant safety and research validity. ${ }^{29}$ Ongoing data analysis cross-checking and monthly discussion in formal 
Figure 3 Study overview.

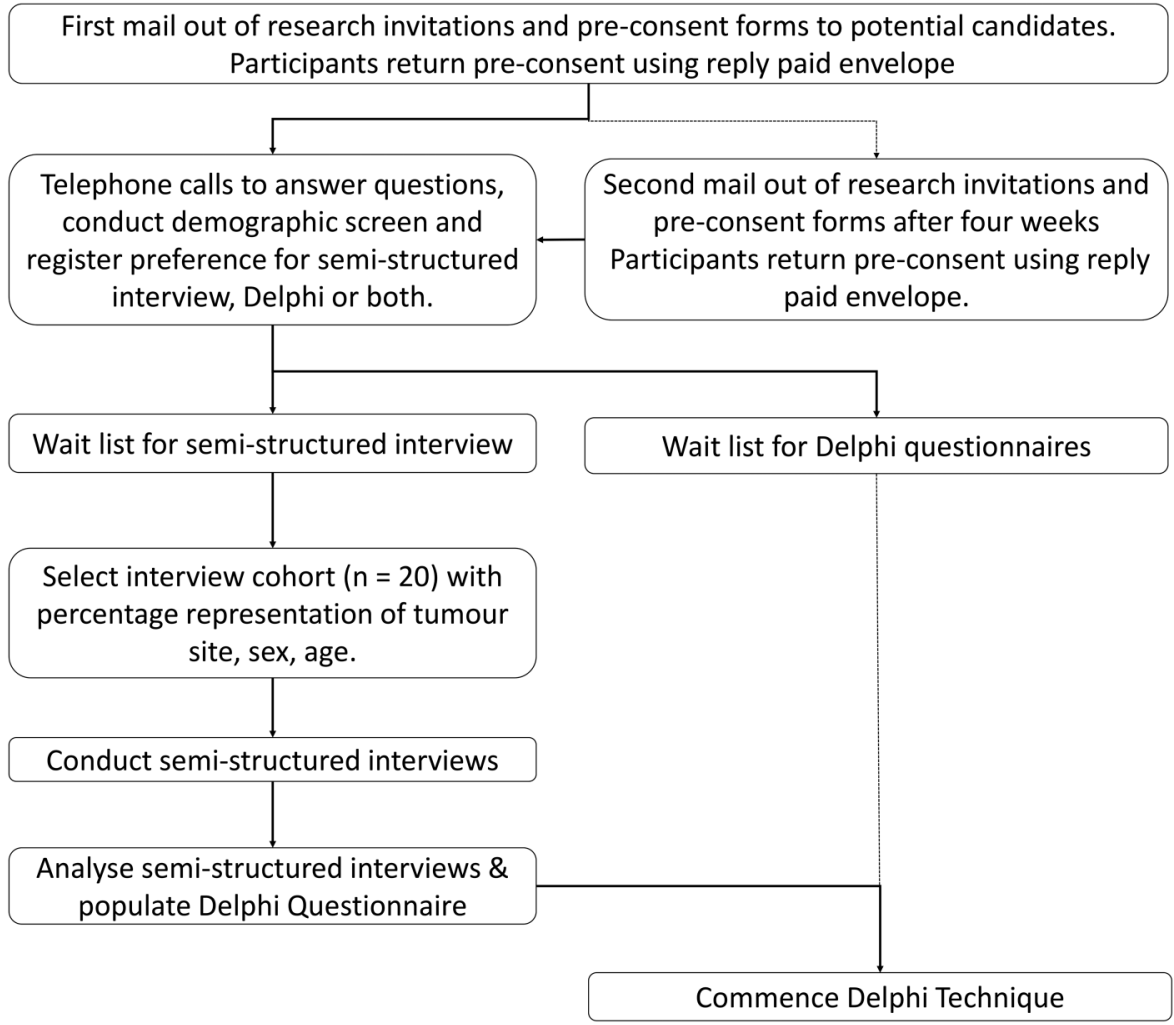

academic supervision with research mentors will provide impartiality to the analysis and help to minimise practitioner bias. $^{33}$

\section{Delphi technique}

The Delphi technique is a multistage process seeking to understand the experiences of individuals by identifying areas of consensus and diversity of opinion on a certain topic, through the use of questionnaires. Statements in the first Delphi questionnaire will be designed based on the themes which emerge from the semistructured interviews. It will be piloted and sent to the research participants for whom a returned consent form is held. The participants will be asked to rate each statement along a five-point Likert Scale, where 0 indicates 'of no importance at all', and 4 indicates 'of absolute importance'. Participants would also be invited to rank, in order of importance, behaviours which typify the features of the therapeutic relationship which they perceive as important to them.

The data analysis following this first questionnaire will look to identify the diversity of opinion, highlight common viewpoints and remove irrelevant data. Participants will be invited to comment on the statements and suggest additional ideas which will also be summarised, analysed and used to generate new or modified statements which will populate the questionnaire for use in the second round.

The second questionnaire will reflect the participants' responses back to them, aiming to understand relationships between statements, clarify topics and start to either build consensus or clarify the scope of diversity in opinions. In order to ensure the quality of the data analysis, consensus will be considered to have been achieved if there is a $>75 \%$ agreement or disagreement for each statement on the questionnaire. ${ }^{34}$

It is expected that there would be at least two rounds in this Delphi, to offer the opportunity to provide feedback to participants, but no more than three rounds. Following guidance provided by Keeney et $a l^{35}$ the investigator will determine the number of rounds from a pragmatic viewpoint considering the time available, the number of rounds required to scope opinion and participant fatigue. Heiko ${ }^{36}$ argues that consensus without stability in responses between the Delphi rounds leaves the results open to misinterpretation. Consensus will be calculated for each round in order to determine the stability of the groups' responses. A third round, if undertaken, will seek to focus on agreement between participants, and stability of consensus or diversity in responses.

\section{Expected outcomes of the study}

Anecdotal evidence from conversations with cancer rehabilitation participants to date would suggest that therapeutic relationships with healthcare providers will be perceived as of a high level of importance. There are a range of other factors which may also be considered important in developing self-management outcomes. This research offers a unique opportunity for the beliefs and opinions of cancer rehabilitation participants to drive service development. 
Table 1 Semistructured interview schedule

\section{Introduction}

Interviewer and interviewee introductions (Read verbatim to interviewee)"This interview will help us to learn more about how rehabilitation works for people affected by cancer. I will ask you questions to help you describe in your own words your thoughts, feelings and beliefs about if/how cancer rehabilitation has helped you to make life changes after cancer. We know that everybody's experience of cancer is different. I want you to feel comfortable enough to say what you really think" Self-determination theory domains Interview prompts

Autonomy

1. Can you tell me how you first came in contact with the cancer rehabilitation service? (eg, Keyworker/doctor/nurse/self-referral)

2. Can you tell me why you came in contact with the cancer rehabilitation service? (eg, symptoms of cancer/treatment or return to life role)

Understanding and expectations of cancer rehabilitation

1. What did you expect from cancer rehabilitation before you had your first assessment? (eg, Get back to normal?/Didn't know what else to do?)

2. Did these expectations change after starting cancer rehabilitation? If so, what made you change? If not, was there a reason?

3. What was the best part about being involved in cancer rehabilitation? (If any)

4. What was the worst part about being involved in cancer rehabilitation? (If any)

1. Has taking part in cancer rehabilitation helped to make changes to your lifestyle or behaviour? (If so, what types of changes have been made? If not, was there a reason?)

2. Can you tell me how important to you it has been to make changes to your lifestyle or behaviour?

3. Can you tell me how confident you feel in maintaining any changes into the future?

Participant perspective of relatedness

1. Can you tell me what you think the rehabilitation team does the best?

2. Can you tell me what the rehabilitation team could do better?

3. Is there anything that the rehabilitation team did or said that stands out in your memory? (eg, special/significant/specific moments that were good or bad)

Importance of relatedness been in making lifestyle or behaviour changes?

2. Many people think of rehabilitation being provided by NHS therapists. Sometimes other practitioners have an important role in your recovery from cancer. Is there anyone else who you feel you have had a significant therapeutic relationship with?

3. Can you describe what it is about this relationship that has helped you/held you back?

\section{Ethical considerations}

This study has been reviewed by the Wales Research Ethics Committee 6. Favourable ethical opinion has been given (15/WA/0331).

\section{DISCUSSION}

There is a growing body of research which has been exploring the effectiveness of cancer rehabilitation programmes. This research has compiled a robust case for the use of physical activity in supporting people to live well with the impact of cancer or cancer treatments. However, there has been little exploration into the relationship between the rehabilitation participants and the professionals delivering the rehabilitation programmes; or the rehabilitation participants' experiences of these programmes.

\section{Strengths and limitations}

Groundwater-Smith and Mockler ${ }^{37}$ propose a framework for ensuring quality of practitioner research. In addition to observing ethical processes, it incorporates demonstrating transparency in processes, being collaborative in nature, being transformative in intent and being able to justify itself to its community or practice. This research protocol has set out to achieve these quality indicators. However, there are limitations to this study which need to be considered.

There is a risk of an overly positive bias in the data, given the existing clinical relationship between participants and the principal investigator. Professional practice doctorates were designed to close the gap between clinical practice and academic knowledge generation by enabling practitioners to approach the solution of realworld problems through researching their own field of practice. ${ }^{38}$ This approach recognises the need to consider practice issues within the complexities of their context, and may limit the generalisability of the findings.

Selecting an external interviewer may have reduced the bias of the pre-existing therapeutic relationship between the participants and the principal investigator. 
However, the decision for the principal investigator to conduct the interviews is congruent with the principles of practitioner research and is closely scrutinised through academic supervision and reflexive journaling. The impact of this relationship will be a point of focus in the analysis and interpretation of results.

Using the Delphi technique offers the advantage of participants' contributions via adding additional ideas and comments, reinforcing the validity of the interview findings. A Delphi technique offers participants a focused structure for providing their thoughts on personal experiences, while remaining anonymous during rounds of consensus building. Being able to reconsider and revise answers in private may also emphasise the reliability of the findings by reducing the risk of group coercion. $^{22}$ Considering the participants in cancer rehabilitation to be the experts in their experience as a group is not only crucial to the design of a Delphi study; it is an opportunity for cancer rehabilitation participants to voice their opinions formally. This will add a unique angle to research into cancer rehabilitation which has been largely researched from a healthcare professional perspective. $^{10}$

Therapeutic relationships have historically been a challenging construct to define. Holmqvist et $a t^{22}$ used a Delphi study to define the therapeutic relationship from a professional perspective. This research aims to build on their work by exploring rehabilitation participants' perceptions of importance of therapeutic relationships, given their role as collaborators in self-management outcome development. ${ }^{27}$ It is not considered likely that this work will be able to identify therapeutic relationships as being effective in isolation from other treatment modalities. Given the focus of this study is to understand the importance, and identify barriers and facilitators, of therapeutic relationships, it is unlikely that the issues of quantifying and subsequently measuring therapeutic relationships will be able to be addressed by this study.

\section{CONCLUSIONS}

The purpose of this study is to better understand how cancer rehabilitation participants perceive the importance of therapeutic relationships, in preference to relying on professional observations. This will enable a foundation for the further development of cancer rehabilitation services. This will aim to ensure future services are fit for purpose, and inform education programmes for professionals working in cancer and non-cancer-specific settings.

\section{Twitter Follow Wendy M Wilkinson @WenWilko}

Acknowledgements The authors are grateful for the support from Maggie's Centre Swansea and Tenovus Cancer Care for providing venues for the semistructured interviews to take place.

Contributors This study was originally conceived by WMW, DF and JR. WMW is responsible for drafting the initial study protocol and data collection. DF and JR have supervised the study and made substantial contribution to the conception, design and analysis involved in the study to date. All authors have agreed on the final version of this article, having been involved in drafting or critically revising draft versions of the manuscript.

Funding The academic fees for this study have been funded by Macmillan Cancer Support Learning and Development Grants (No 420; No 1811; No 2048); Learning and Development support from Abertawe Bro Morgannwg University Health Board and Golau Cancer Charity and a teaching bursary from the School of Human and Health Sciences at the Swansea University. The BMJ Open Access publication charge was funded by the Wales Cancer Network.

Competing interests This study will be carried out as part of the principal investigator's candidature for a professional practice doctorate, and will make a significant contribution to the thesis for this award. The study runs parallel to the principal investigator's employment in a cancer rehabilitation service. It is sponsored by the Research and Development Team in Abertawe Bro Morgannwg University Health Board. The authors declare that they have no conflict of interest.

Ethics approval Wales Research Ethics Committee 6.

Provenance and peer review Not commissioned; externally peer reviewed.

Open Access This is an Open Access article distributed in accordance with the Creative Commons Attribution Non Commercial (CC BY-NC 4.0) license, which permits others to distribute, remix, adapt, build upon this work noncommercially, and license their derivative works on different terms, provided the original work is properly cited and the use is non-commercial. See: http:// creativecommons.org/licenses/by-nc/4.0/

\section{REFERENCES}

1. CRUK. Cancer incidence in the UK in 2011. Cancer Research UK, 2014. http://publications.cancerresearchuk.org/downloads/Product/ CS_REPORT_INCIDENCE.pdf

2. CRUK. Key facts all cancers combined: how common is cancer? 2014. http://publications.cancerresearchuk.org/publicationformat/ formatfactsheet/keyfactsall.html

3. DH, Macmillan Cancer Support, NHS Improvement. Living with \& beyond cancer. Taking Action to Improve Outcomes UK, 2013.

4. Craig P, Dieppe P, Macintyre S, et al., Medical Research Council Guidance. Developing and evaluating complex interventions: the new Medical Research Council guidance. BMJ 2008;337:a1655.

5. Korstjens I, May AM, van Weert E, et al. Quality of life after self-management cancer rehabilitation: a randomized controlled trial comparing physical and cognitive-behavioral training versus physical training. Psychosom Med 2008;70:422-9.

6. Pearson EJ, Twigg VJ. A framework for rehabilitation for cancer survivors. Eur J Cancer Care (Engl) 2013;22:701-8.

7. Oldervoll LM, Kaasa S, Hjermstad MJ, et al. Physical exercise results in the improved subjective well-being of a few or is effective rehabilitation for all cancer patients? Eur J Cancer 2004;40: 951-62.

8. van Weert E, May AM, Korstjens I, et al. Cancer-related fatigue and rehabilitation: a randomized controlled multicenter trial comparing physical training combined with cognitive-behavioral therapy with physical training only and with no intervention. Phys Ther 2010;90:1413-25.

9. Mewes JC, Steuten LM, ljzerman MJ, et al. Effectiveness of multidimensional cancer survivor rehabilitation and cost-effectiveness of cancer rehabilitation in general: a systematic review. Oncologist 2012;17:1581-93.

10. Scott DA, Mills M, Black A, et al. Multidimensional rehabilitation programmes for adult cancer survivors. Cochrane Database Syst Rev 2013;(3):CD007730.

11. Silver JK, Baima J. Cancer prehabilitation: an opportunity to decrease treatment-related morbidity, increase cancer treatment options, and improve physical and psychological health outcomes. Am J Phys Med Rehabil 2013;92:715-27.

12. Li C, Carli F, Lee L, et al. Impact of a trimodal prehabilitation program on functional recovery after colorectal cancer surgery: a pilot study. Surg Endosc 2013;27:1072-82.

13. Morris GS, Gallagher GH, Baxter MF, et al. Pulmonary rehabilitation improves functional status in oncology patients. Arch Phys Med Rehabil 2009;90:837-41.

14. van Weert E, Hoekstra-Weebers JE, May AM, et al. The development of an evidence-based physical self-management 
rehabilitation programme for cancer survivors. Patient Educ Couns 2008:71:169-90.

15. Foster $\mathrm{C}$, Breckons $\mathrm{M}$, Cotterell $\mathrm{P}$, et al. Cancer survivors' self-efficacy to self-manage in the year following primary treatment. J Cancer Surviv 2015;9:11-19.

16. The Health Foundation. http://www.health.org.uk/sites/health/files/ HelpingPeopleHelpThemselves.pdf (accessed 1 Mar 2016).

17. McCorkle R, Ercolano E, Lazenby M, et al. Self-management: enabling and empowering patients living with cancer as a chronic illness. CA Cancer J Clin 2011;61:50-62.

18. Jones F, Livingstone E, Hawkes L. 'Getting the balance between encouragement and taking over': reflections on using a new stroke self-management programme. Physiother Res Int 2013;18:91-9.

19. Hill HC, Paley J, Forbat L. Observations of professional-patient relationships: a mixed-methods study exploring whether familiarity is a condition for nurses' provision of psychosocial support. Palliat Med 2014;28:256-63.

20. Horvath $A O$, Luborsky $L$. The role of the therapeutic alliance in psychotherapy. J Consult Clin Psychol 1993;61:561-73.

21. Hall AM, Ferreira PH, Maher CG, et al. The influence of the therapist-patient relationship on treatment outcome in physical rehabilitation: a systematic review. Phys Ther 2010;90:1099-110.

22. Holmqvist $K$, Holmefur M, Ivarsson AB. Therapeutic use of self as defined by Swedish occupational therapists working with clients with cognitive impairments following acquired brain injury: a Delphi study. Aust Occup Ther J 2013;60:48-55.

23. Finlay L. The practice of psychosocial occupational therapy. 3rd edn Cheltenham: Stanley Thornes, 2004

24. Schnur JB, Montgomery GH. A systematic review of therapeutic alliance, group cohesion, empathy, and goal consensus/ collaboration in psychotherapeutic interventions in cancer: uncommon factors? Clin Psychol Rev 2010;30:238-47.

25. Ryan RM, Patrick H, Deci EL, et al. Facilitating health behaviour change and its maintenance: interventions based on self-determination theory. Eur Health Psychol 2008;10:2-5.

26. $\mathrm{Ng}$ JY, Ntoumanis $\mathrm{N}$, Thøgersen-Ntoumani $\mathrm{C}$, et al. Self-determination theory applied to health contexts: a meta-analysis. Perspect Psychol Sci 2012;7:325-40.
27. Boger E, Ellis J, Latter S, et al. Self-management and self-management support outcomes: a systematic review and mixed research synthesis of stakeholder views. PLOS ONE 2015;10:e0130990.

28. Quality Health. Wales Cancer Patient Experience Survey: National Report 2013. Published January 2014. Welsh Government, Macmillan Cancer Support and NHS Wales. http://gov.wales/docs/ dhss/publications/140117canceren.pdf (accessed 25 Feb 2016).

29. Kvale S. InterViews: an introduction to qualitative research interviewing. SAGE Publications, 1996.

30. Graneheim UH, Lundman B. Qualitative content analysis in nursing research: concepts, procedures and measures to achieve trustworthiness. Nurse Educ Today 2004;24:105-12.

31. Sandelowski M. Focus on research methods. Whatever happened to qualitative description? Res Nurs Health 2000;23:334-40.

32. Stanley M, Nayar S. Deepening understandings. In: Nayar S, Stanley MD, eds. Qualitative research methodologies for occupational science and therapy. Abingdon, Oxon: Routledge, 2015:8-20.

33. Stanley M. Qualitative descriptive: A very good place to start. In: Nayar S, Stanley MD, eds. Qualitative research methodologies for occupational science and therapy. Abingdon,Oxon: Routledge, 2015:21-36.

34. Diamond IR, Grant RC, Feldman BM, et al. Defining consensus: a systematic review recommends methodologic criteria for reporting of Delphi studies. J Clin Epidemiol 2014;67:401-9.

35. Keeney S, Hasson F, McKenna H. Consulting the oracle: ten lessons from using the Delphi technique in nursing research. $J A d v$ Nurs 2006;53:205-12.

36. Heiko A. Consensus measurement in Delphi studies: review and implications for future quality assurance. Technol Forecast Soc Change 2012;79:1525-36.

37. Groundwater-Smith S, Mockler N. Ethics in practitioner research: an issue of quality. Research Papers in Education, 2007. (Special Issue on Research Quality).

38. Fulton J, Kuit J, Sanders G, et al. The role of the Professional Doctorate in developing professional practice. J Nurs Manag 2012;20:130-9. 